\title{
RLKs orchestrate the signaling in plant male-female interaction
}

\author{
Hongju $\mathrm{Li}^{*} \&$ Wei-Cai Yang ${ }^{* *}$ \\ State Key Laboratory of Molecular Developmental Biology, Institute of Genetics and Developmental Biology, Chinese Academy of Sciences, \\ Beijing 100101, China
}

Received May 7, 2016; accepted May 16, 2016; published online August 12, 2016

\begin{abstract}
Different from animals, sessile plants are equipped with a large receptor-like kinase (RLK) superfamily. RLKs are a family of single trans-membrane proteins with divergent $\mathrm{N}$-terminal extracellular domains capped by a signal peptide and C-terminal intracellular kinase. Researches in the last two decades have uncovered an increasing number of RLKs that regulate plant development, stress response and sexual reproduction, highlighting a dominant role of RLK signaling in cell-to-cell communications. Sexual reproduction in flowering plants is featured by interactions between the male gametophyte and the female tissues to facilitate sperm delivery and fertilization. Emerging evidences suggest that RLKs regulate almost every aspect of plant reproductive process, especially during pollination. Therefore, in this review we will focus mainly on the function and signaling of RLKs in plant male-female interaction and discuss the future prospects on these topics.
\end{abstract}

RLK, signaling, plant reproduction, pollen tube, female gametophyte, male-female interaction, pollen tube growth and guidance, pollen tube reception

Citation: Li, H., and Yang, W.C. (2016). RLKs orchestrate the signaling in plant male-female interaction. Sci China Life Sci 59, 867-877. doi: $10.1007 / \mathrm{s} 11427-016-0118-\mathrm{x}$

\section{RLKS AND PLANT REPRODUCTION: A BRIEF INTRODUCTION}

Ligands-activated RLKs regulate a wide range of biological processes in plants, including vegetative and reproductive development, and pathogenic response. The activation mode to some extent is similar to the RLKs in axon guidance, cell assembly or migration of animal cells (Kullander and Klein, 2002). RLKs perceive and transmit extracellular signals, such as chemical molecules and peptides, into the cell through phosphorylation thereby activating a series of downstream components. Understanding the diversity and specificity of RLK signaling requires knowledge of the ligands, the activation mode and the downstream signaling cascade in the cellular context. Arabidopsis and rice genomes encode more than 600 and 1000 RLK members, re-

*Corresponding author (email: hjli@genetics.ac.cn)

**Corresponding author (email: wcyang@genetics.ac.cn) spectively (Shiu and Bleecker, 2003; Gish and Clark, 2011). A large percentage of these RLKs have not been functionally identified and only in a few cases the cognate ligands or the signaling cascades have been elucidated. One major obstacle of the functional analysis is functional redundancy caused by genome expansion. The RLKs in Arabidopsis consist of a number of subfamilies with different extracellular domains which are the major determinant of the signaling specificities (Shiu and Bleecker, 2003). Recent progress highlights the key roles of peptide signals and RLKs in sexual reproduction in flowering plants.

Flowering plants employ a delicate mating system to ensure its high prosperity on the earth. One remarkable feature is that pollen tube (male gametophyte), a tip-growing tubular structure, is evolved to deliver the immobile sperm cells in its cytoplasm traversing the female tissues to the female gametophyte (embryo sac). Successful fertilization relies on sequential intercellular interactions between pollen tubes and sporophytic tissues including stigma, style, transmitting 
tract, as well as the embryo sac (Figure 1). In the compatible female tissues, the elongating pollen tube is promoted and supported by female-derived signals and nutrients and finally attracted to the embryo sac. Another typical feature of flowering plants is that the multicellular embryo sac is wrapped in the ovule which in turn is embedded in the ovary. The representative embryo sac is composed of two synergids and an egg cell at the micropyle, a large central cell in the middle, and three antipodal cells at the chalazal pole (Yang et al., 2010). The embryo sac secretes attractants to guide the entry of pollen tubes (Higashiyama and Takeuchi, 2015), which is to some extent similar to axon guidance in animals (Palanivelu and Preuss, 2000). Upon compatible interaction with the female gametophyte, the pollen tube bursts in the receptive synergids to release the two sperm cells which subsequently fuse with the egg and the central cell, respectively, to accomplish double fertilization. During the guided and receptive processes, intercellular interactions take place between the pollen tube and female tissues or the embryo sac, in which secreted peptides and RLKs play important roles. Here, we will discuss our current understanding of RLKs and the signaling components in male-female interaction. Peptide signaling in pollen tube guidance has been nicely reviewed recently (Kanaoka and Higashiyama, 2015; Qu et al., 2015).

\section{RLKS IN POLLEN-STIGMA INTERACTION}

Cell-to-cell signaling between pollen and stigma sets the beginning of pollination. More than half of the flowering plants utilize self-incompatibility (SI) systems to prevent inbreeding and enforce out-crossing. In Brassicaceae, SI is genetically controlled by variants of the single highly polymorphic genetic locus, called the S-locus (Suzuki et al., 1999; Takasaki et al., 2000). Each functional S-locus variant comprises the $\mathrm{S}$ haplotype, containing an S-locus receptor kinase (SRK) and an S-locus cysteine-rich protein (SCR) gene. SRK is a transmembrane serine/threonine kinase acting as the female determinant expressed on the stigma epidermis (Figure 2). The extracellular domain of SRK contains a S-domain including two lectin domains, a EGF-like and PAN_APPLE domains (Ivanov et al., 2010). The male determinant SCR $(\sim 6 \mathrm{kD})$ is a defensin-like peptide derived mainly from the tapetal cells of the anther (Shiba et al., 2001). SRK perceives SCR deposited on the pollen coat to initiate sporophytic SI response during incompatible pollination (Takasaki et al., 2000; Takayama et al., 2001). SI is only initiated when the pollen land on the "self" stigma of the same haplotype to block pollen hydration and germination which leads to rejection of "self" pollen to prohibit self-pollination (Kachroo et al., 2001). During out-crossing or selfing in the self-compatible species, like Arabidopsis thaliana in which the SRK or SCR alleles are non-functional, the non-self SCR could not activate SRK, thus the pollen rejection signaling is not triggered.

Sequence analysis suggests that SRK and SCR coevolve and display high sequence divergence between variants (Sato et al., 2002). All the SCR variants exhibit the same 3D structure, but share less than $50 \%$ sequence similarity at amino acid level (Nasrallah and Nasrallah, 2014). The specificity of the SRK-SCR pair is implied to be determined by a few amino acids while the underlying principle is still

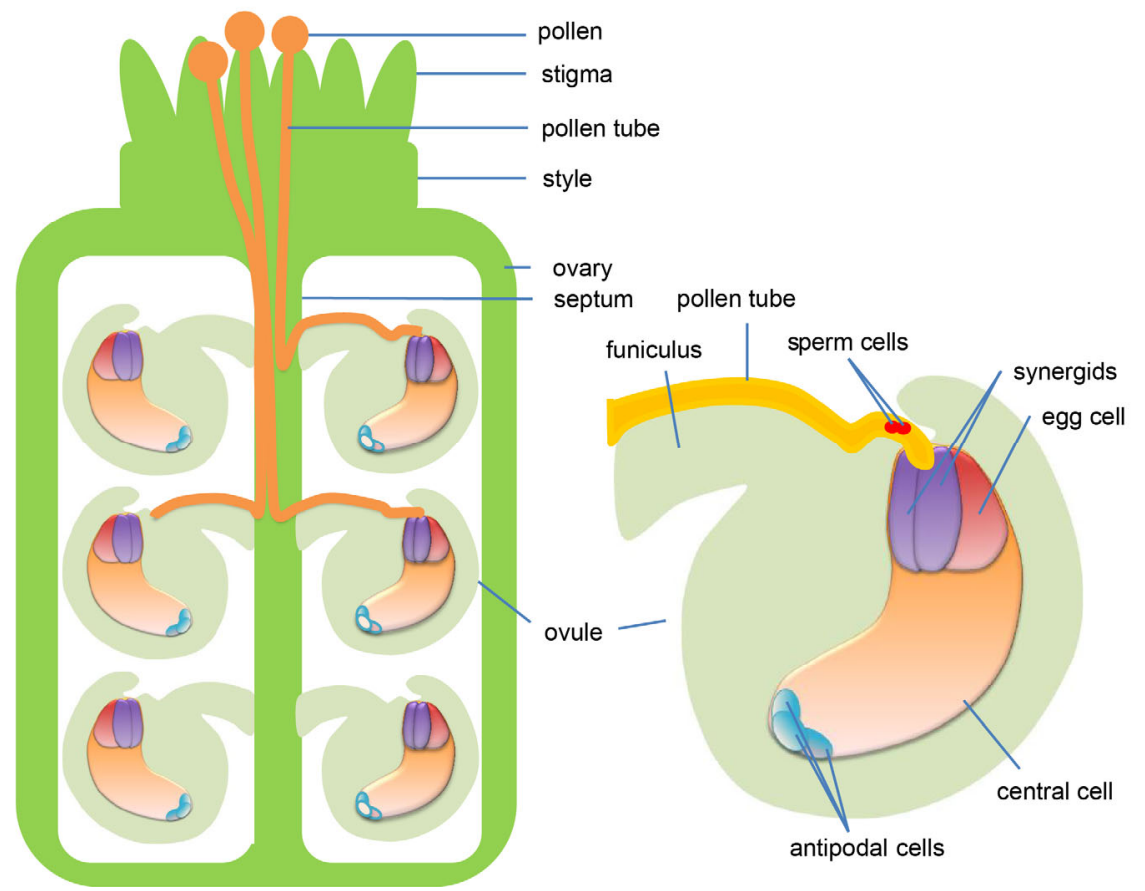

Figure 1 Schematic representation of pollination and fertilization processes in flowering plants. 


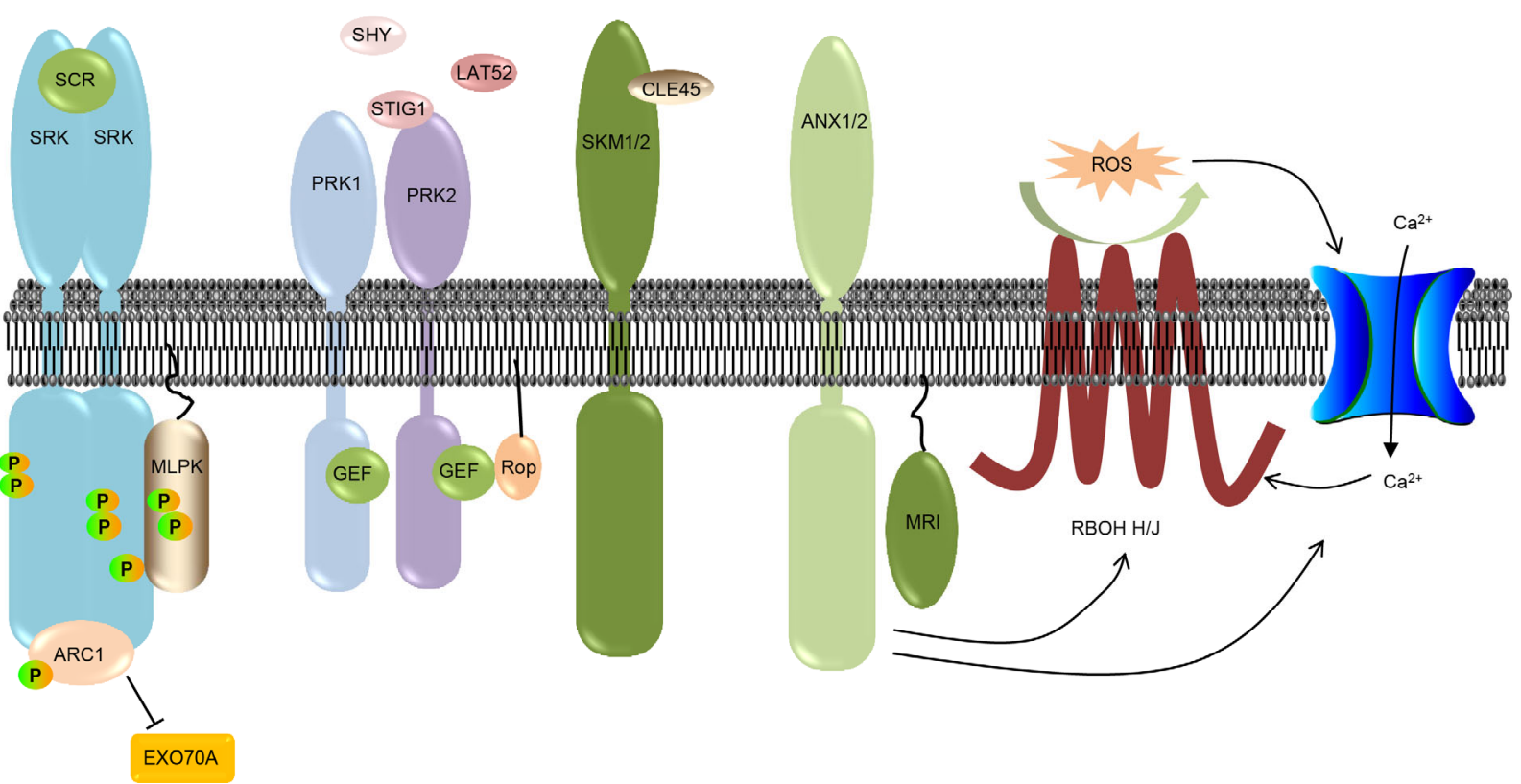

Figure 2 Models of the RLK signaling in different steps of pollen tube-pistil interaction. For simplicity, all RLKs are drawn on the same plasma membrane.

unclear. Transferring the SRK-SCR pair of A. lyrata to A. thaliana confers the SI response (Nasrallah et al., 2002), indicating that the ligand-receptor is the primary determinant of SI. This raises the question whether the cellular signaling cascades are generally shared among species.

The signaling cascade triggered on the stigma epidermal cell by SCR-activated SRK is still poorly understood. Through genetic and biochemical approaches, two proteins were found to interact with SRK: the membrane-anchored cytoplasmic kinase MLPK (M locus protein kinase) (Murase et al., 2004) and the E3 ligase ARC1 (ARM repeat-containing protein 1) (Gu et al., 1998; Stone et al., 1999, 2003). EXO70A1, a component of the exocyst complex, interacts with ARC1 and is proposed to enhance the secretion of substances essential for pollen hydration. MLPK and SRK undergo phosphorylation upon SI pollination and they can phosphorylate ARC1 which possibly target EXO70A1 for degradation and subsequently inhibit secretion by the stigma (Figure 2). However, functional test of these three components in transgenic SCR-SRK incompatible A. thaliana suggests that the homologs of EXO70A1, MLPK and ARC1, are not involved in the recombinant SI system (Kitashiba et al., 2011). The molecular basis of this discrepancy is unclear. Up to now, only limited numbers of SCR-SRK signaling components are functionally identified and the function of candidate interacting factors is still unclear. In A. thaliana, with the loss of SRK function, the relieved selection pressure on the downstream components might cause their functional diversification. Thus, two scenarios could be conceived that either multiple signal cas- cades or different cascades are utilized in other species.

\section{RLKS MEDIATE POLLEN TUBE GROWTH IN PISTIL}

After compatible pollen-stigma interaction, peptides and their paired RLKs sustain the invasive pollen tube growth. Pollen-specific receptor kinases (PRKs) compose a family of pollen-specific leucine-rich repeat (LRR) subtype of RLKs in diverse plant species. LePRK1 and LePRK2 in tomato pollen bind stably with each other and dissociate when exposed to the pistil extracts (Wengier et al., 2003). The pollen-derived peptide ligand LAT52 is a cysteine-rich peptide (CRP) with four cysteines and binds the extracellular domain of LePRK2 before pollination, and this binding is replaced by the stigma-derived LeSTIG1 (stigma-specific protein 1) (Tang et al., 2002, 2004). In petunia, the pollen cell wall located SHY binds the extracellular domain of PRK2, and likely performs a similar role as LAT52. Knock-down of LAT52 or SHY impairs pollen performance on the stigma, indicating that both LAT52 and SHY contribute to optimum reproductive success (Muschietti et al., 1994; Guyon et al., 2004). LeSTIG1 was recently found to bind phosphatidylinositol 3-phosphate $\left(\mathrm{PI}_{3} \mathrm{P}\right)$ and induces pollen tube growth in vitro (Huang et al., 2014). Although how the pollen-derived LAT52 and SHY and the stigma-derived STIG1 coordinate pollen tube performance on the stigma through LePRK1-PRK2 remains to be established, it appears that different ligands function in different stages during pollination. 
Different ligands determine the different signaling modes of the RLKs (Figure 2). Phosphorylation of LePRK2 is essential for LePRK1-PRK2 dimer formation. The style-derived factor STIL induces dephosphorylation of LePRK2, thus disrupts its association with LePRK1 (Wengier et al., 2010). This dynamic phosphorylation appears to be correlated with different ligands, while its molecular basis still needs further investigation especially how phosphorylation sites are selected in response to specific ligands. Consistently, LePRK2 exists as multiple isoforms and is phosphorylated at two motifs in the juxtamembrane domain and these phosphorylation act antagonistically during pollen tube growth (Salem et al., 2010). This antagonistic regulation confers a delicate fine-tuning of PRK signaling capacity, thereby transducing different extracellular signals into the pollen tube. Pollen grains over-expressing LePRK1 germinate pollen tubes with enlarged tips, often with "bleb" generating from the tip, which suggests a role of LePRK1 in pollen tube growth (Gui et al., 2014). This finding confirms that the dissociated LePRK1 and LePRK2 both function during pollen tube growth within the pistil, possibly in response to different ligands. On the contrary, another aspect making the signaling pathway more complex is that PRKs share overlapping function as evidenced by the aggravated shortened pollen tubes in mutants depleted with multiple PRKs (Chang et al., 2012).

Interestingly, both LePRK1 and LePRK2 function through KPP (kinase partner protein), a Rop (Rho-like small GTPase from plant) guanine nucleotide exchange factor (RopGEF) (Zhang et al., 2008; Gui et al., 2014). In Arabidopsis, several members of PRK homologs also regulate pollen tube growth through RopGEF (Zhang and McCormick, 2007; Chang et al., 2012). Similar to the effect of excessive LePRK2 or KPP, over-expression of AtPRK2 or AtGEF causes ectopic GEF activity at a broad range on the plasma membrane of the tube tip, leading to pollen tube depolarization. Thus the mechanism of PRK2-RopGEF mediated pathway is pivotal for pollen tube polarity. This polarized growth is self-organized through positive feedback regulation by spatiotemporal modulation of Rop activity (Yalovsky et al., 2008). The spatial distribution of RopGEF and the negative regulator RopGAP restrict Rop activity at the tube tip to maintain the oscillatory tip growth. Considering the expression of several GEFs and multiple PRK members and their multiple complex isoforms in pollen tubes, the signaling specificity from RLKs to GEFs or the biological significance of this signaling multiplicity have not been determined. Although the PRK2-RopGEF signaling module has long been established, several key questions to understand the PRK-mediated signaling have not been answered: (i) What is the ligand of each PRK? (ii) How the ligand-binding on the extracellular domain activate the cytoplasmic kinase? (iii) How the phosphorylation state of the cytoplasmic domain of PRK coordinates GEF activation?

RLKs also function in pollen tube growth under stress condition. SKM1 (sterility-regulating kinase member 1) and SKM2 are two homologous LRR-RLKs expressed in the pollen which perceive the pistil-expressed CLE45, a CLV3/ESR-related peptides in Arabidopsis (Endo et al., 2013). Interestingly, CLE45 expression is induced in the transmitting tract at high temperature. Disturbance of CLE45-SKM1/SKM2 signaling reduces pollen tube growth in the pistil at high temperature. This finding reveals a protecting mechanism of RLKs in adapted pollen-pistil interaction at hostile environment.

\section{RLKS REGULATE THE INTEGRITY OF POLLEN TUBES}

Pollen tubes have to coordinate cell wall dynamics with internal growth machinery to maintain fast growth and cell integrity. ANX1 and ANX2, two RLKs of CrRLK1L family, is shown to regulate pollen tube integrity (Figure 2). The CrRLK1L family is conserved in plants and consists of 17 members in Arabidopsis. ANXs are expressed in pollen tubes and loss-of-function of both $A N X 1$ and $A N X 2$ causes precocious pollen tube burst when grown in vitro or in the pistil (Boisson-Dernier et al., 2009; Miyazaki et al., 2009). Recently, a plasma membrane-localized receptor-like cytoplasmic kinase MARIS (MRI) is identified as a positive regulator of the ANX pathway. Similarly, mri mutants display spontaneous pollen tube rupture and the $M R I^{R 240 C}$ point mutation suppresses pollen tube rupture defect of anx1 anx2 (Boisson-Dernier et al., 2015). Cytosolic kinase can transduce the signal from RLK down to different components, while the physical link between ANX and MRI and the signaling cascade of kinases are still unclear. Reactive oxygen species (ROS) has been established to be involved in a wide range of cellular signaling events (Figure 2). Two NADPH oxidases, RbohH and RbohJ, functioning in ROS generation, act downstream of ANX and fine tune $\mathrm{Ca}^{2+}$ homeostasis (Boisson-Dernier et al., 2013). Both ANX and ROS signaling promote the exocytosis pathway which subsequently influences the cell wall thickness. RbohD is activated via the direct phosphorylation by cytosolic kinase in the RLK complex during pathogen response (Li et al., 2014), it would be plausible to speculate that MRI or other cytosolic kinases also activate RbohH/J. It was proposed that ANX and the CrRLK1L family, as a signaling integrator, could sense the cell wall integrity simultaneously (Cheung and $\mathrm{Wu}, 2011$; Lindner et al., 2012). The CrRLK1L family contains two malectin-like domains at the extracellular region sharing limited homology with the animal endoplasmic reticulum-localized malectin that bind di-glucose (Schallus et al., 2008). This sequence similarity is indicative of a cell wall binding potential of the family. Another CrRLK1L member THE1 suppresses plant growth under cellulose deficiency, which further indicates that this family could mediate a cell wall-related process (Hematy et al., 2007). But the mechanical link between these RLKs and the cell wall 
material is still unclear. Considering that the extracellular matrix of plant cells is highly heterogeneous, the dissection of direct binding of the proteins to the cell wall is difficult. CrRLK1L member has been shown to bind secreted peptide as well (Haruta et al., 2014). Thus the identification of the ligand of ANX is also of great interest to understand the signaling activation mechanism. On the other hand, cell wall stiffness is dynamically fine-tuned by the balanced activity between pectin methylesterase (PME) and pectin methylesterase inhibitor (PMEI), which regulate the homeostasis of pectin (Jiang et al., 2005; Rockel et al., 2008). Homogalacturonan is the major pectin species, and is synthesized in the Golgi and secreted in methyl-esterified form at the pollen tube tip where it is further demethylesterified by PME synergistically with the growth oscillation (Wolf and Greiner, 2012). PMEI counters the enzyme activity of PME through direct binding (Hothorn et al., 2004). Given that $\mathrm{PME}$ activity has an alkaline $\mathrm{pH}$ preference, $\mathrm{H}^{+}$release accompanied by the removal of methyl groups leads to PME inhibition. In future studies, the elucidation of the molecular relationship between ANX signaling and the pectin state will help to understand how cell wall dynamics is regulated by the RLK signaling.

Additionally, the $\mathrm{Ca}^{2+}$ influx disruption caused by the loss of calcium permeable channel CNGC18 also causes pollen tube rupture similar to that of anxl anx2 (Frietsch et al., 2007). Interestingly, it was observed that the rupture of the anxl anx 2 pollen tubes is accompanied by a transient high $\mathrm{Ca}^{2+}$ spike, while in $\mathrm{rboh} \mathrm{j} / \mathrm{h}$ pollen tubes, the $\mathrm{Ca}^{2+}$ is decreased and less stable (Boisson-Dernier et al., 2013). Furthermore, mechanical stress, most likely, also plays a role in regulating the pollen tube integrity. It was reported that the loss-of-function mutation in the pollen-expressed mechanosensitive channel MSL 8 causes pollen tube rupture upon germination (Hamilton et al., 2015). Surprisingly, MSL8 forms a mechanosensitive ion channel with a preference for anions when expressed in Xenopus laevis oocytes. It will be of interest to dissect the intersection or relationship between ANX and these cation and anion channels.

\section{RLKS IN POLLEN TUBE GUIDANCE}

After navigation in the style and transmitting tract, pollen tubes penetrate the septum surface and climb onto the funiculus to find the ovules. This process is designated as funicular guidance (Figure 1). Genetic evidence from mutants with either embryo sac and/or integument developmental defects shows discrepancy as to the source of funiculus guidance cues. Brassica pollen tube can penetrate the style and grow in the transmitting tract of Arabidopsis, but unable to penetrate the septum, i.e. not attracted by the ovule-derived funicular guidance cues (Kandasamy et al., 1994). This phenomenon suggests that the signal should be diffusible secreted proteins or peptides with species specificity. Phytosulfokine (PSK) is a disulfated pentapeptide and functions as a universal peptide growth factor in plants. PSK plays a role in the attraction of pollen tubes at funiculus stage (Stuhrwohldt et al., 2015), while its attracting activity still awaits to be demonstrated. PSKR, the receptor of PSK, regulates cell expansion in plant development (Matsubayashi et al., 2002). Loss of PSKR or TPST which sulfates PSK, causes arrested pollen tube growth in the pistil with reduced funicular guidance (Stuhrwohldt et al., 2015). This phenotype is pronounced when the mutant was used as the male or female side in cross-pollination or under self-pollination, indicating a more complex mechanism. We found recently that SERK family acts as the co-receptor of PSKR (Wang et al., 2015). However, it is still unclear whether the SERK family is also involved in pollen tube performance or other similar RLK family function as the co-receptor of PSKR during pollination. Two mitogen-activated protein kinases, MPK3 and MPK6, are also required for funicular guidance of pollen tubes in Arabidopsis (Guan et al., 2014).

After finding the ovule, pollen tubes continue to grow through the micropylar opening to find the embryo sac, a process called micropylar guidance (Figures 1 and 3). During micropylar guidance, the defensin-like peptides LUREs secreted from the synergids attract pollen tubes in both Arabidopsis and Torenia fournieri (Okuda et al., 2009; Takeuchi and Higashiyama, 2012). In maize, a distinct peptide ZmEA1 emitted from the embryo sac serves as the female attractant (Márton et al., 2005). These findings suggest that the monocot and dicot may use different peptide signals to attract pollen tubes. Plasma membrane localized receptors in the pollen tubes have been implied to perceive the guidance cues from the embryo sac (Li et al., 2011; Li and Yang, 2012). Recently, two independent works identified the putative LURE1 receptors in Arabidopsis (Wang et al., 2016, Takeuchi and Higashiyama, 2016). Two RLKs, MIK1/2 and MDIS1, serve as the receptor/co-receptor complex to perceive LURE1 and transduce the signal into the cell by transphosphorylation (Wang et al., 2016). PRK6, a member of the PRK family mentioned above, was also shown to be essential for both LURE1 perception and pollen tube growth (Takeuchi and Higashiyama, 2016). These findings raise two possibilities: there is more than one receptor for a ligand or they are among the multiple components of one signaling pathway (Cheung and Wu, 2016).

Ligand-induced dimerization, co-receptor requirement and phosphorylation are important mechanisms to activate the RLK signaling cascades. A number of RLKs employ co-receptors to transduce the ligand signal into the cell, such as FLS2-BAK1 and EFR-BAK1 co-receptor complex perceiving pathogen-derived peptide flg22 and elf18 respectively in innate immunity (Roux et al., 2011), BRI1-BAK1 receptor complex perceiving brassinosteroid during plant growth, as well as PSKR1-SERK (Santiago et al., 2013). In previous reported receptor/co-receptor complex in plants, the co-receptor does not directly participate in ligand bind- 
ing, but recruited to the ligand-receptor complex to transduce the signal by transphosphorylation (Santiago et al., 2013; Sun et al., 2013; Wang et al., 2015). In contrast, both MIK1/2 and MDIS1 bind LURE1, although with different affinity (Wang et al., 2016). The constitutive interaction between MDIS1 and MIK1 possibly enables a quick and efficient sensing of the gradient of female attractants along the growing path of the pollen tube. Although the biological significance of the phosphorylation of MDIS1 by MIK1 induced by LURE1 is still unclear, it is possible that LURE1 induces conformational change of the kinase domain to facilitate phosphorylation and activation of the downstream proteins. Site-directed mutagenesis and structural analysis of MDIS1-MIKs complex will improve our understanding of the biochemical mechanism of the MDIS1-MIK1 sensing of LURE1.

Both receptor-mediated phosphorylation and RopGEF-based ROP activation function in cell surface signaling in pollen tubes. The discovery of MDIS1-MIK1 complex highlights the critical role of ligand-induced receptor phosphorylation, which may trigger a phosphorylation cascade of downstream pathways critical for guided pollen tube growth. Indeed, the cytosolic kinases LIP1 (lost in pollen tube guidance 1) and LIP2 are required for pollen tube response to LURE1 (Liu et al., 2013) and interacts with PRK6 (Takeuchi and Higashiyama, 2016). Additionally, AGC kinases and MPK3/6 cascade also play a role in regulating pollen tube growth and guidance (Zhang et al., 2009). Alternatively, ROP signaling is also implied to play a role in guidance through the finding that PRK6 interacts with RopGEF12 (Takeuchi and Higashiyama, 2016). It is not known whether this interaction leads to RopGEF12 phosphorylation. Intriguingly, the kinase activity of PRK6 is not required for the pollen tube perception of LURE1 but essential for the pollen tube growth (Takeuchi and Higashiyama, 2016). Previous studies shows that GEF1 phosphorylation by PRK2 was essential for its full function (Chang et al., 2012). It raises the query that if the kinase-dependent GEF activation is common for all PRK family member or just specific to PRK2. LePRK2 undergoes two different phosphorylation modes which antagonistically regulate its function during pollen tube growth. Thus, whether PRK6 itself or GEF12 needs to be phosphorylated by other RLKs, for example MIK1, is an interesting question. And our unpublished data suggest that MDIS1/MIK signaling may also include GEF pathway. Further investigation is needed to clarify the relationship between the MIK1/2-MDIS1 and PRK6 signaling pathways. The LURE1-mediated guidance is a redirecting process of the tip growth. This suggests that LURE1 perception initiates a new signal cascade that redistributes the cytoskeleton and vesicles to the LURE perception site on the plasma membrane. Further dissection of the link between the GEF-ROP and phosphorylation pathway is of importance to understand mechanisms coordinating this redirecting process.

\section{RLKs in pollen tube reception}

Upon arrival at the embryo sac, the pollen tube tip interacts directly with the synergids of the embryo sac. This intercellular interaction is called pollen tube reception that is also regulated by RLKs (Figure 3). FERONIA (FER), a homologue of ANX, is the first RLK found to regulate pollen tube reception in the synergid. Pollen tubes fail to rupture in the synergid of the fer mutant ovules, resulting in overgrowth of the pollen tube inside the embryo sac (Huck et al., 2003; Escobar-Restrepo et al., 2007). This suggests that FER is required to trigger pollen tube rupture. Interestingly, FER is expressed broadly except in pollen, which correlates with its multiple roles in plants. Indeed, FER plays roles in seedling development, root hair growth, trichome morphogenesis, responses to hormone and pathogen. The function of FER and CrRLK1L family has been discussed by several excellent reviews (Boisson-Dernier et al., 2011; Cheung and $\mathrm{Wu}, 2011$; Lindner et al., 2012). Here we will mainly focus on its role in pollen tube reception.

FER is accumulated at the plasma membrane of the synergid and the function is dependent on the extracellular signals. It was proposed that FER on the synergid membrane perceives signals from the pollen tube to initiate programed cell death of the interacting synergid, and in turn, the tube also sense signals from the synergid to trigger pollen tube rupture. The reproductive proteins involved in fertilization or pollination generally display sequence polymorphism. Sequence analysis suggests that the extracellular domain of FER was under positive selection during species evolution, thus the signal perception of FER could function as an interspecies cross barrier (Escobar-Restrepo et al., 2007). In contrast, the cytoplasmic kinase domain of FER can be replaced with that of ANX for full function of FER (Kessler et al., 2015). Although the ligand of FER from the pollen tube is not yet identified, studies in plant growth have unveiled that a secreted peptide-RALF1 (rapid ALkalization factor 1), most likely acts as the ligand of FER during seedling growth (Haruta et al., 2014). RALF family has been linked to cell wall alkalization and inhibits cell expansion in many plant species (Matsubayashi, 2014). Interestingly, a pollen-specific RALF has been reported to inhibit pollen tube elongation in tomato (Solanum lycopersicum) (Covey et al., 2010). Arabidopsis genome encodes 34 RALF members and some of them are expressed in pollen and/or synergids or induced by pollination, suggesting a role in gametophyte interaction or function although none has been functionally characterized (Wuest et al., 2010; Boavida et al., 2011). The pollen tube rupture is preceded by a temporal growth arrest of the pollen tube upon contact with the synergid, which is essential for the following burst. Considering the role in restraining cell expansion, RAFL from the synergid might inhibit the pollen tube growth at the synergid. Interestingly, triple mutant of three transcription factors MYB97, MYB101 and MYB120 which are expressed in the 


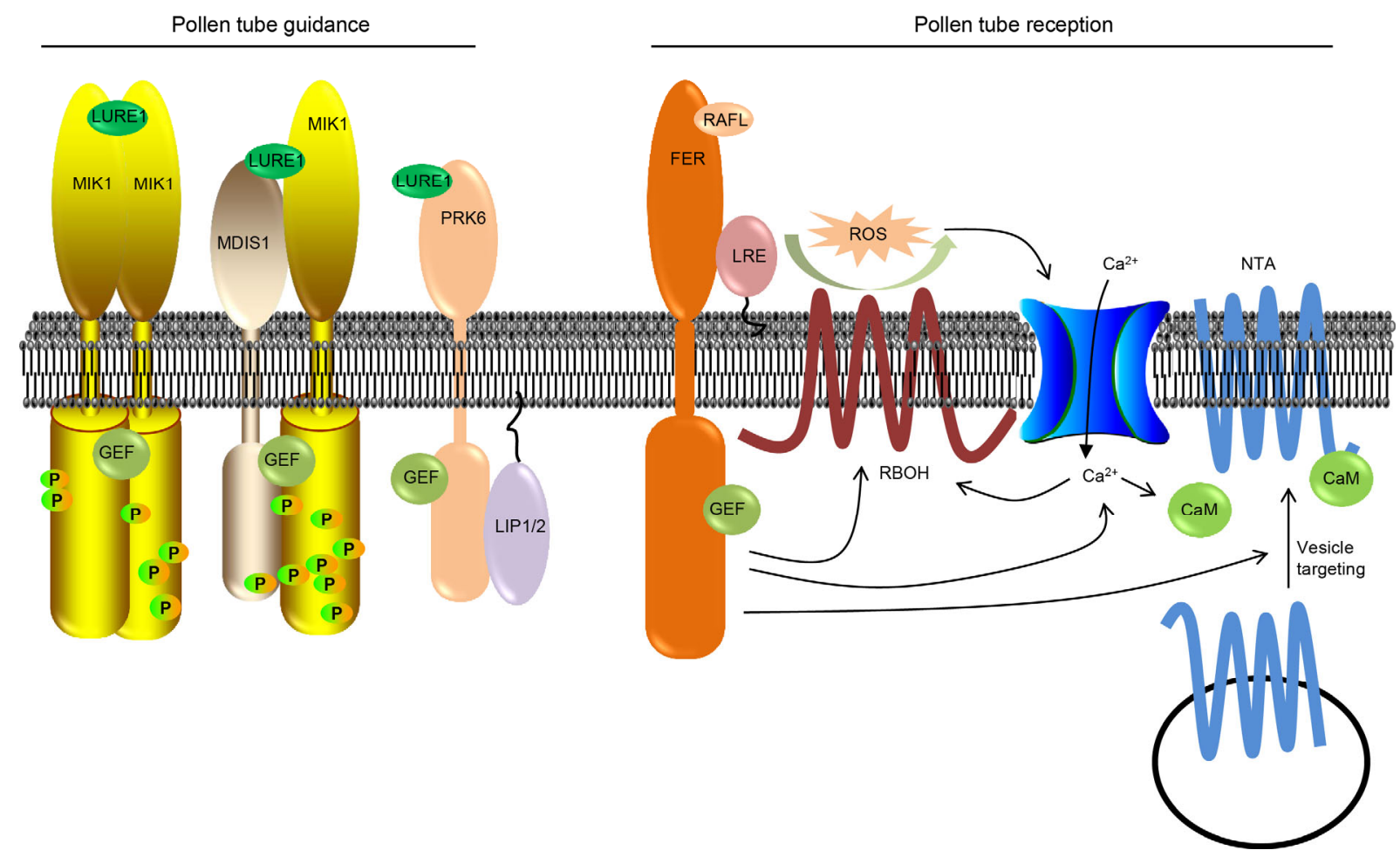

Figure 3 Schematic representation of RLK signaling during pollen tube guidance and reception.

pollen tube displays fer-like phenotype (Leydon et al., 2013; Liang et al., 2013), suggesting that they may mediate the production of FER ligand in the tube. ANX1/2 in the pollen tube is required to prevent the tube from rupture. It is not known how the homologous RLKs perform antagonistic roles with FER promoting and ANX preventing pollen tube burst. Undoubtedly, the identification of ligands for FER and ANX would provide more insight into the underlying mechanism.

LORELEI (LRE) is a glycosylphosphatidylinositol (GPI)-anchored protein (GPI-APs) expressed in the synergid and required for pollen tube reception. GPI-APs are important cell sensors in eukaryotic cells, especially in sperm-egg fusion in animals, and they are often localized in the lipid raft on the plasma membrane (Stein et al., 2004). The GPI synthetic pathway and several members of the GPI-APs have been reported to function in plant reproduction (Lalanne et al., 2004; Tsukamoto et al., 2010; Li et al., 2013; Dai et al., 2014). Recent evidences show that the GPI anchor is dispensable for the LRE function and even ectopically expressed LRE from the pollen tubes could non-cell-autonomously rescues lre mutant (Liu et al., 2016). LRE and its homolog LLG1 have been reported to interact with FER and function as a component of the FER-regulated Rho GTPase signaling pathway in plant growth ( $\mathrm{Li}$ et al., 2015). Thus it was postulated that LRE and LLG1 function as co-receptor or chaperone of FER.

Recent data show that FER transduces signals through $\mathrm{Ca}^{2+}$ and ROS. One of the outstanding downstream compo- nents of FER is $\mathrm{Ca}^{2+}$, which is a universal second messenger in multiple cellular responses. Transient increase of cytosolic $\mathrm{Ca}^{2+}$ induced by RALF1 is reduced in fer seedlings (Haruta et al., 2014). Similarly, the pollen tube arrival at the synergid is also accompanied by a characteristic FER-mediated $\mathrm{Ca}^{2+}$ responses (Iwano et al., 2012; Ngo et al., 2014). Pollen tube arrival triggers sequential cytoplasmic $\mathrm{Ca}^{2+}$ response including an initial $\mathrm{Ca}^{2+}$ spike and the following $\mathrm{Ca}^{2+}$ flood in the receptive synergid, which precedes a prolonged high level of $\mathrm{Ca}^{2+}$ accompanied by the rupture and the collapse of the receptive synergid. In the synergid of fer and lre, this pollen tube-triggered $\mathrm{Ca}^{2+} \mathrm{dy}-$ namics is dramatically compromised (Ngo et al., 2014). These suggest that such $\mathrm{Ca}^{2+}$ signature is specific to pollen tube reception. Another key signaling molecule downstream of FER is ROS. In the synergid, ROS accumulates at the filiform apparatus, a structure with membrane protrusion from the synergids and is required for pollen tube rupture (Duan et al., 2014). This ROS accumulation is dependent on FER and LRE in a $\mathrm{Ca}^{2+}$ dependent pathway. It is still unknown if additional ROS burst takes place upon pollen tube arrival. In this context, it is not known whether the ROS generation is dependent on the ligand perception. In addition, $\mathrm{ROS}$ and $\mathrm{Ca}^{2+}$ mutually regulate each other in vegetative tissues (Gilroy et al., 2014; Gorlach et al., 2015). How they cross-talk is not clear during pollen tube reception.

An exciting finding is that FER is required for mechanical signaling by regulating $\left[\mathrm{Ca}^{2+}\right]_{\mathrm{cyt}}$ and $\mathrm{pH}$ response (Shih et al., 2014). In fer roots, but not in other mutants of CrRLK 
members, the biphasic $\mathrm{Ca}^{2+}$ and $\mathrm{pH}$ response under stretch is impaired and only the first $\mathrm{Ca}^{2+}$ response remains. Based on these studies, FER is proposed to monitor the growth-related strain to balance the dynamics of cell expansion which drives the internal mechanical strain and $\mathrm{Ca}^{2+}$ fluctuation. How FER perceives the stretch and translates the strain to intracellular $\mathrm{pH}$ and $\mathrm{Ca}^{2+}$ change remains unknown. Similarly, in the receptive synergid of fer, the first $\mathrm{Ca}^{2+}$ response is remained although prolonged but the second phase is trailed off (Ngo et al., 2014). This suggests the possibility that FER specifically regulates the second $\mathrm{Ca}^{2+}$ response which correlates with the mechanical gating of $\mathrm{Ca}^{2+}$ channels in the synergid. This second phase $\mathrm{Ca}^{2+}$ flood is proposed to be the direct trigger for the cell death of the receptive synergid and pollen tube burst. This speculation is rational considering the durative growth of the tube to the synergid after initial cell surface contact. The functional characterization of mechanosensitive ion channels expressed in the synergid will shed light on the molecular mechanism of FER on $\mathrm{Ca}^{2+}$ modulation. Based on these findings, in FER mediated pollen tube reception, both ROS generation and $\mathrm{Ca}^{2+}$ entry in the embryo sac are indispensable for pollen tube burst. However, in ANX1 mediated pollen tube-autoregulation, the reduced ROS and $\mathrm{Ca}^{2+}$ appear to be correlated with pollen tube burst. This discrepancy still lacks reasonable explanations supported by evidences, but the exogenous and endogenous ROS might act differently. RALF1-FER interaction induces the phosphorylation of plasma membrane AHA2 $\left(\mathrm{H}^{+}\right.$-adenosine triphosphatase 2) (Haruta et al., 2014), which may contribute to the FER-mediated $\mathrm{pH}$ response upon stretch. AHA-mediated $\mathrm{pH}$ homeostasis may also contribute to cell wall mechanics and membrane potential which in turn may influence the $\mathrm{Ca}^{2+}$ and ROS signaling.

Another downstream component of FER is NORTIA (NTA) which encodes seven-transmembrane protein previously designated MLO7. NTA is expressed specifically in the synergid and upon pollen tube arrival it is targeted micropylarly to the synergid membrane in a FER-dependent manner (Kessler et al., 2010). Interestingly, FER is also involved in fungal invasion in a similar mode with MLO in barley, tomato and Arabidopsis (Piffanelli et al., 2004; Consonni et al., 2006; Bai et al., 2008). This indicates that fertilization and pathogen response share some common signaling components during evolution. In barley, the $\mathrm{Ca}^{2+}$-dependent binding of calmodulin to MLO is required for the function of MLO, suggesting a $\mathrm{Ca}^{2+}$-mediated signaling pathway (Kim et al., 2002). Interestingly, the magnitude of $\mathrm{Ca}^{2+}$ response is reduced, but the pattern is not changed in the nta synergids with defective pollen tube reception. Thus NTA is a modulator of the magnitude of $\mathrm{Ca}^{2+}$ signature (Ngo et al., 2014). These imply a possible feedback regulation between NTA and $\mathrm{Ca}^{2+}$, although the dependence of NTA on $\mathrm{Ca}^{2+}$ or calmodulin has not been demonstrated. Together, the FER-mediated membrane tar- geting of NTA and its role in pollen tube reception need further investigation in the future.

Furthermore, FER might also act through RAC/ROP signaling pathway by interacting with multiple RopGEFs (Duan et al., 2010), as that of PRKs-mediated pathway during pollen tube growth. It is unclear if GEF-ROP module is also recruited by FER in the synergid or ANX in the pollen tube. FER depletion leads to root hair rupture resembling the precocious bursting of anxl anx2 pollen tubes, raising the paradigm that FER/CrRLK1L are among the central regulators of cell polar growth or cell expansion in general. Taken together, a complex network including, at least if not all, extracellular ligand perception, ROS production, $\mathrm{pH}$ homeostasis, $\mathrm{Ca}^{2+}$ dynamics and cell wall integration, are employed during pollen tube reception.

\section{PROSPECTS}

The expression profiling results showed that a plenty of RLKs and secreted peptides were expressed in pollen tubes, ovules and pistils, indicating an intense male-female communication through ligand-RLK signaling (Chae and Lord, 2011; Loraine et al., 2013; Qu et al., 2015). To date, only a few RLK-ligand pairs have been established or functionally analyzed. Thus, one of the major tasks in the future is to identify more RLK-ligand pairs and decipher their roles during plant reproduction. We anticipate that future studies on the functional characterization and dissection of signaling pathways mediated by RLKs in the interaction between pollen tube and different female tissues will expand and deepen our understanding of signaling mechanisms in plant reproduction.

Compliance and ethics The author(s) declare that they have no conflict of interest.

Acknowledgements This work was financially supported by the Ministry of Science and Technology of China (2013CB945103), and the National Natural Science Foundation of China (31330053).

Bai, Y., Pavan, S., Zheng, Z., Zappel, N.F., Reinstadler, A., Lotti, C., De Giovanni, C., Ricciardi, L., Lindhout, P., Visser, R., Theres, K., and Panstruga, R. (2008). Naturally occurring broad-spectrum powdery mildew resistance in a central American tomato accession is caused by loss of mlo function. Mol Plant Microbe Interact 21, 30-39.

Boavida, L.C., Borges, F., Becker, J.D., and Feijo, J.A. (2011). Whole genome analysis of gene expression reveals coordinated activation of signaling and metabolic pathways during pollen-pistil interactions in Arabidopsis. Plant Physiol 155, 2066-2080.

Boisson-Dernier, A., Kessler, S.A., and Grossniklaus, U. (2011). The walls have ears: the role of plant CrRLK1Ls in sensing and transducing extracellular signals. J Exp Bot 62, 1581-1591.

Boisson-Dernier, A., Franck, C.M., Lituiev, D.S., and Grossniklaus, U. (2015). Receptor-like cytoplasmic kinase MARIS functions downstream of CrRLK1L-dependent signaling during tip growth. Proc Natl Acad Sci USA 112, 12211-12216.

Boisson-Dernier, A., Lituiev, D.S., Nestorova, A., Franck, C.M., Thirugnanarajah, S., and Grossniklaus, U. (2013). ANXUR 
receptor-like kinases coordinate cell wall integrity with growth at the pollen tube tip via NADPH oxidases. PLoS Biol 11, e1001719.

Boisson-Dernier, A., Roy, S., Kritsas, K., Grobei, M.A., Jaciubek, M., Schroeder, J.I., and Grossniklaus, U. (2009). Disruption of the pollen-expressed FERONIA homologs ANXUR1 and ANXUR2 triggers pollen tube discharge. Development 136, 3279-3288.

Chae, K., and Lord, E.M. (2011). Pollen tube growth and guidance: roles of small, secreted proteins. Ann Bot 108, 627-636.

Chang, F., Gu, Y., Ma, H., and Yang, Z. (2012). AtPRK2 promotes ROP1 activation via RopGEFs in the control of polarized pollen tube growth. Mol Plant 6, 1187-1201.

Cheung, A.Y., and Wu, H.M. (2011). THESEUS 1, FERONIA and relatives: a family of cell wall-sensing receptor kinases? Curr Opin Plant Biol 14, 632-641.

Cheung, A.Y., and Wu, H.M. (2016). Plant biology: LURE is bait for multiple receptors. Nature 531, 178-180.

Consonni, C., Humphry, M.E., Hartmann, H.A., Livaja, M., Durner, J., Westphal, L., Vogel, J., Lipka, V., Kemmerling, B., Schulze-Lefert, P., Somerville, S.C., and Panstruga, R. (2006). Conserved requirement for a plant host cell protein in powdery mildew pathogenesis. Nat Genet $38,716-720$

Covey, P.A., Subbaiah, C.C., Parsons, R.L., Pearce, G., Lay, F.T., Anderson, M.A., Ryan, C.A., and Bedinger, P.A. (2010). A pollen-specific RALF from tomato that regulates pollen tube elongation. Plant Physiol 153, 703-715.

Dai, X.R., Gao, X.Q., Chen, G.H., Tang, L.L., Wang, H., and Zhang, X.S. (2014). ABNORMAL POLLEN TUBE GUIDANCE1, an endoplasmic reticulum-localized mannosyltransferase homolog of GLYCOSYLPHOSPHATIDYLINOSITOL10 in yeast and PHOSPHATIDYLINOSITOL GLYCAN ANCHOR BIOSYNTHESIS B in human, is required for Arabidopsis pollen tube micropylar guidance and embryo development. Plant Physiol 165, 1544-1556.

Duan, Q., Kita, D., Li, C., Cheung, A.Y., and Wu, H.M. (2010). FERONIA receptor-like kinase regulates RHO GTPase signaling of root hair development. Proc Natl Acad Sci USA 107, 17821-17826.

Duan, Q., Kita, D., Johnson, E.A., Aggarwal, M., Gates, L., Wu, H.M., and Cheung, A.Y. (2014). Reactive oxygen species mediate pollen tube rupture to release sperm for fertilization in Arabidopsis. Nat Commun $5,3129$.

Endo, S., Shinohara, H., Matsubayashi, Y., and Fukuda, H. (2013). A novel pollen-pistil interaction conferring high-temperature tolerance during reproduction via CLE45 signaling. Curr Biol 23, 1670-1676.

Escobar-Restrepo, J.M., Huck, N., Kessler, S., Gagliardini, V., Gheyselinck, J., Yang, W.C., and Grossniklaus, U. (2007). The FERONIA receptor-like kinase mediates male-female interactions during pollen tube reception. Science 317, 656-660.

Frietsch, S., Wang, Y.F., Sladek, C., Poulsen, L.R., Romanowsky, S.M., Schroeder, J.I., and Harper, J.F. (2007). A cyclic nucleotide-gated channel is essential for polarized tip growth of pollen. Proc Natl Acad Sci USA 104, 14531-14536.

Gilroy, S., Suzuki, N., Miller, G., Choi, W.G., Toyota, M., Devireddy, A.R., and Mittler, R. (2014). A tidal wave of signals: calcium and ROS at the forefront of rapid systemic signaling. Trends Plant Sci 19, 623-630.

Gish, L.A., and Clark, S.E. (2011). The RLK/Pelle family of kinases. Plant J 66, 117-127.

Gorlach, A., Bertram, K., Hudecova, S., and Krizanova, O. (2015). Calcium and ROS: a mutual interplay. Redox Biol 6, 260-271.

Gu, T., Mazzurco, M., Sulaman, W., Matias, D.D., and Goring, D.R. (1998). Binding of an arm repeat protein to the kinase domain of the S-locus receptor kinase. Proc Natl Acad Sci USA 95, 382-387.

Guan, Y., Lu, J., Xu, J., McClure, B., and Zhang, S. (2014). Two mitogen-activated protein kinases, MPK3 and MPK6, are required for funicular guidance of pollen tubes in Arabidopsis. Plant Physiol 165, 528-533.

Gui, C.-P., Dong, X., Liu, H.-K., Huang, W.-J., Zhang, D., Wang, S.-J., Barberini, M.L., Gao, X.-Y., Muschietti, J., McCormick, S., and Tang, W.-H. (2014). Overexpression of the tomato pollen receptor kinase LePRK1 rewires pollen tube growth to a blebbing mode. Plant Cell 26,
$3538-3555$

Guyon, V., Tang, W.H., Monti, M.M., Raiola, A., Lorenzo, G.D., McCormick, S., and Taylor, L.P. (2004). Antisense phenotypes reveal a role for SHY, a pollen-specific leucine-rich repeat protein, in pollen tube growth. Plant J 39, 643-654.

Hamilton, E.S., Jensen, G.S., Maksaev, G., Katims, A., Sherp, A.M., and Haswell, E.S. (2015). Mechanosensitive channel MSL8 regulates osmotic forces during pollen hydration and germination. Science 350, 438-441.

Haruta, M., Sabat, G., Stecker, K., Minkoff, B.B., and Sussman, M.R. (2014). A peptide hormone and its receptor protein kinase regulate plant cell expansion. Science 343, 408-411.

Hematy, K., Sado, P.E., Van Tuinen, A., Rochange, S., Desnos, T., Balzergue, S., Pelletier, S., Renou, J.P., and Hofte, H. (2007). A receptor-like kinase mediates the response of Arabidopsis cells to the inhibition of cellulose synthesis. Curr Biol 17, 922-931.

Higashiyama, T., and Takeuchi, H. (2015). The mechanism and key molecules involved in pollen tube guidance. Annu Rev Plant Biol 66, 393-413.

Hothorn, M., Wolf, S., Aloy, P., Greiner, S., and Scheffzek, K. (2004). Structural insights into the target specificity of plant invertase and pectin methylesterase inhibitory proteins. Plant Cell 16, 3437-3447.

Huang, W.J., Liu, H.K., McCormick, S., and Tang, W.H. (2014). Tomato pistil factor STIG1 promotes in vivo pollen tube growth by binding to phosphatidylinositol 3-phosphate and the extracellular domain of the pollen receptor kinase LePRK2. Plant Cell 26, 2505-2523.

Huck, N., Moore, J.M., Federer, M., and Grossniklaus, U. (2003). The Arabidopsis mutant feronia disrupts the female gametophytic control of pollen tube reception. Development 130, 2149-2159.

Ivanov, R., Fobis-Loisy, I., and Gaude, T. (2010). When no means no: guide to Brassicaceae self-incompatibility. Trends Plant Sci 15, 387-394.

Iwano, M., Ngo, Q.A., Entani, T., Shiba, H., Nagai, T., Miyawaki, A., Isogai, A., Grossniklaus, U., and Takayama, S. (2012). Cytoplasmic $\mathrm{Ca}^{2+}$ changes dynamically during the interaction of the pollen tube with synergid cells. Development 139, 4202-4209.

Jiang, L., Yang, S.L., Xie, L.F., Puah, C.S., Zhang, X.Q., Yang, W.C., Sundaresan, V., and Ye, D. (2005). VANGUARD1 encodes a pectin methylesterase that enhances pollen tube growth in the Arabidopsis style and transmitting tract. Plant Cell 17, 584-596.

Jingjing Liu, S.Z., Xinyang Guo, Lihong Hao,, Xiaolin Wei, Q.H., Yingnan Hou, Jiao Shi,, and Chaoyang Wang, H.G., and Li-Jia Qu. (2013). Membrane-bound RLCKs LIP1 and LIP2 are essential male factors controlling male-female attraction in Arabidopsis. Curr Biol 23, $1-6$.

Kachroo, A., Schopfer, C.R., Nasrallah, M.E., and Nasrallah, J.B. (2001). Allele-specific receptor-ligand interactions in Brassica self-incompatibility. Science 293, 1824-1826.

Kanaoka, M.M., and Higashiyama, T. (2015). Peptide signaling in pollen tube guidance. Curr Opin Plant Biol 28, 127-136.

Kandasamy, M.K., Nasrallah, J.B., and Nasrallah, M.E. (1994). Pollen-pistil interactions and developmental regulation of pollen tube growth in Arabidopsis. Development 120, 3405-3418.

Kessler, S.A., Lindner, H., Jones, D.S., and Grossniklaus, U. (2015). Functional analysis of related CrRLK1L receptor-like kinases in pollen tube reception. EMBO Rep 16, 107-115.

Kessler, S.A., Shimosato-Asano, H., Keinath, N.F., Wuest, S.E., Ingram, G., Panstruga, R., and Grossniklaus, U. (2010). Conserved molecular components for pollen tube reception and fungal invasion. Science 330, 968-971.

Kim, M.C., Panstruga, R., Elliott, C., Muller, J., Devoto, A., Yoon, H.W., Park, H.C., Cho, M.J., and Schulze-Lefert, P. (2002). Calmodulin interacts with MLO protein to regulate defence against mildew in barley. Nature 416, 447-451.

Kitashiba, H., Liu, P., Nishio, T., Nasrallah, J.B., and Nasrallah, M.E. (2011). Functional test of Brassica self-incompatibility modifiers in Arabidopsis thaliana. Proc Natl Acad Sci USA 108, 18173-18178.

Kullander, K., and Klein, R. (2002). Mechanisms and functions of Eph and ephrin signalling. Nat Rev Mol Cell Biol 3, 475-486. 
Lalanne, E., Honys, D., Johnson, A., Borner, G.H., Lilley, K.S., Dupree, P., Grossniklaus, U., and Twell, D. (2004). SETH1 and SETH2, two components of the glycosylphosphatidylinositol anchor biosynthetic pathway, are required for pollen germination and tube growth in Arabidopsis. Plant Cell 16, 229-240.

Leydon, A.R., Beale, K.M., Woroniecka, K., Castner, E., Chen, J., Horgan, C., Palanivelu, R., and Johnson, M.A. (2013). Three MYB transcription factors control pollen tube differentiation required for sperm release. Curr Biol 23, 1209-1214.

Li, C., Yeh, F.L., Cheung, A.Y., Duan, Q., Kita, D., Liu, M.C., Maman, J., Luu, E.J., Wu, B.W., Gates, L., Jalal, M., Kwong, A., Carpenter, H., and $\mathrm{Wu}, \mathrm{H} . \mathrm{M}$. (2015). Glycosylphosphatidylinositol-anchored proteins as chaperones and co-receptors for FERONIA receptor kinase signaling in Arabidopsis. eLife 4, 4.

Li, H.J., and Yang, W.C. (2012). Emerging role of ER quality control in plant cell signal perception. Protein Cell 3, 10-16.

Li, H.J., Xue, Y., Jia, D.J., Wang, T., Hi, D.Q., Liu, J., Cui, F., Xie, Q., Ye, D., and Yang, W.C. (2011). POD1 regulates pollen tube guidance in response to micropylar female signaling and acts in early embryo patterning in Arabidopsis. Plant Cell 23, 3288-3302.

Li, L., Li, M., Yu, L., Zhou, Z., Liang, X., Liu, Z., Cai, G., Gao, L., Zhang, X., Wang, Y., Chen, S., and Zhou, J.M. (2014). The FLS2-associated kinase BIK1 directly phosphorylates the NADPH oxidase RbohD to control plant immunity. Cell Host Microbe 15, 329-338.

Li, S., Ge, F.R., Xu, M., Zhao, X.Y., Huang, G.Q., Zhou, L.Z., Wang, J.G., Kombrink, A., McCormick, S., Zhang, X.S., and Zhang, Y. (2013). Arabidopsis COBRA-LIKE 10, a GPI-anchored protein, mediates directional growth of pollen tubes. Plant J 74, 486-497.

Liang, Y., Tan, Z.M., Zhu, L., Niu, Q.K., Zhou, J.J., Li, M., Chen, L.Q., Zhang, X.Q., and Ye, D. (2013). MYB97, MYB101 and MYB120 function as male factors that control pollen tube-synergid interaction in Arabidopsis thaliana fertilization. PLoS Genet 9, e1003933.

Lindner, H., Muller, L.M., Boisson-Dernier, A., and Grossniklaus, U. (2012). CrRLK1L receptor-like kinases: not just another brick in the wall. Curr Opin Plant Biol 15, 659-669.

Liu, X., Castro, C.A., Wang, Y., Noble, J.A., Ponvert, N.D., Bundy, M.G., Hoel, C.R., Shpak, E.D., and Palanivelu, R. (2016). The role of LORELEI in pollen tube reception at the interface of the synergid cell and pollen tube requires the modified eight-cysteine motif and the receptor-like kinase FERONIA. Plant Cell pii: tpc.00703.2015.

Loraine, A.E., McCormick, S., Estrada, A., Patel, K., and Qin, P. (2013). RNA-seq of Arabidopsis pollen uncovers novel transcription and alternative splicing. Plant Physiol 162, 1092-1109.

Márton, M.L., Cordts, S., Broadhvest, J., and Dresselhaus, T. (2005). Micropylar pollen tube guidance by egg apparatus 1 of maize. Science 307, 573-576.

Matsubayashi, Y. (2014). Posttranslationally modified small-peptide signals in plants. Annu Rev Plant Biol 65, 385-413.

Matsubayashi, Y., Ogawa, M., Morita, A., and Sakagami, Y. (2002). An LRR receptor kinase involved in perception of a peptide plant hormone, phytosulfokine. Science 296, 1470-1472.

Miyazaki, S., Murata, T., Sakurai-Ozato, N., Kubo, M., Demura, T., Fukuda, H., and Hasebe, M. (2009). ANXUR1 and 2, sister genes to FERONIA/SIRENE, are male factors for coordinated fertilization. Curr Biol 19, 1327-1331.

Murase, K., Shiba, H., Iwano, M., Che, F.S., Watanabe, M., Isogai, A., and Takayama, S. (2004). A membrane-anchored protein kinase involved in Brassica self-incompatibility signaling. Science 303, 1516-1519.

Muschietti, J., Dircks, L., Vancanneyt, G., and McCormick, S. (1994). LAT52 protein is essential for tomato pollen development: pollen expressing antisense LAT52 RNA hydrates and germinates abnormally and cannot achieve fertilization. Plant J 6, 321-338.

Nasrallah, J.B., and Nasrallah, M.E. (2014). S-locus receptor kinase signalling. Biochem Soc Trans 42, 313-319.

Nasrallah, M.E., Liu, P., and Nasrallah, J.B. (2002). Generation of self-incompatible Arabidopsis thaliana by transfer of two S locus genes from A. lyrata. Science 297, 247-249.

Ngo, Q.A., Vogler, H., Lituiev, D.S., Nestorova, A., and Grossniklaus, U. (2014). A calcium dialog mediated by the FERONIA signal transduction pathway controls plant sperm delivery. Dev Cell 29, 491-500.

Okuda, S., Tsutsui, H., Shiina, K., Sprunck, S., Takeuchi, H., Yui, R., Kasahara, R.D., Hamamura, Y., Mizukami, A., Susaki, D., Kawano, N., Sakakibara, T., Namiki, S., Itoh, K., Otsuka, K., Matsuzaki, M., Nozaki, H., Kuroiwa, T., Nakano, A., Kanaoka, M.M., Dresselhaus, T., Sasaki, N., and Higashiyama, T. (2009). Defensin-like polypeptide LUREs are pollen tube attractants secreted from synergid cells. Nature 458, 357-361.

Palanivelu, R., and Preuss, D. (2000). Pollen tube targeting and axon guidance: parallels in tip growth mechanisms. Trends Cell Biol 10, 517-524.

Piffanelli, P., Ramsay, L., Waugh, R., Benabdelmouna, A., D’Hont, A., Hollricher, K., Jorgensen, J.H., Schulze-Lefert, P., and Panstruga, R. (2004). A barley cultivation-associated polymorphism conveys resistance to powdery mildew. Nature 430, 887-891.

Qu, L.J., Li, L., Lan, Z., and Dresselhaus, T. (2015). Peptide signalling during the pollen tube journey and double fertilization. J Exp Bot 66, 5139-5150.

Rockel, N., Wolf, S., Kost, B., Rausch, T., and Greiner, S. (2008). Elaborate spatial patterning of cell-wall PME and PMEI at the pollen tube tip involves PMEI endocytosis, and reflects the distribution of esterified and de-esterified pectins. Plant J 53, 133-143.

Roux, M., Schwessinger, B., Albrecht, C., Chinchilla, D., Jones, A., Holton, N., Malinovsky, F.G., Tor, M., de Vries, S., and Zipfel, C. (2011). The Arabidopsis leucine-rich repeat receptor-like kinases BAK1/SERK3 and BKK1/SERK4 are required for innate immunity to hemibiotrophic and biotrophic pathogens. Plant Cell 23, 2440-2455.

Salem, T., Mazzella, A., Barberini, M.L., Wengier, D., Motillo, V., Parisi, G., and Muschietti, J. (2010). Mutations in two putative phosphorylation motifs in the tomato pollen receptor kinase LePRK2 show antagonistic effects on pollen tube length. J Biol Chem 286, 4882-4891.

Santiago, J., Henzler, C., and Hothorn, M. (2013). Molecular mechanism for plant steroid receptor activation by somatic embryogenesis co-receptor kinases. Science 341, 889-892.

Sato, K., Nishio, T., Kimura, R., Kusaba, M., Suzuki, T., Hatakeyama, K., Ockendon, D.J., and Satta, Y. (2002). Coevolution of the S-locus genes SRK, SLG and SP11/SCR in Brassica oleracea and B. rapa. Genetics 162, 931-940.

Schallus, T., Jaeckh, C., Feher, K., Palma, A.S., Liu, Y., Simpson, J.C., Mackeen, M., Stier, G., Gibson, T.J., Feizi, T., Pieler, T., and Muhle-Goll, C. (2008). Malectin: a novel carbohydrate-binding protein of the endoplasmic reticulum and a candidate player in the early steps of protein N-glycosylation. Mol Biol Cell 19, 3404-3414.

Shiba, H., Takayama, S., Iwano, M., Shimosato, H., Funato, M., Nakagawa, T., Che, F.S., Suzuki, G., Watanabe, M., Hinata, K., and Isogai, A. (2001). A pollen coat protein, SP11/SCR, determines the pollen S-specificity in the self-incompatibility of Brassica species. Plant Physiol 125, 2095-2103.

Shih, H.W., Miller, N.D., Dai, C., Spalding, E.P., and Monshausen, G.B. (2014). The receptor-like kinase FERONIA is required for mechanical signal transduction in Arabidopsis seedlings. Curr Biol 24, 1887-1892.

Shiu, S.H., and Bleecker, A.B. (2003). Expansion of the receptor-like kinase/Pelle gene family and receptor-like proteins in Arabidopsis. Plant Physiol 132, 530-543.

Stein, K.K., Primakoff, P., and Myles, D. (2004). Sperm-egg fusion: events at the plasma membrane. J Cell Sci 117, 6269-6274.

Stone, S.L., Arnoldo, M., and Goring, D.R. (1999). A breakdown of Brassica self-incompatibility in ARC1 antisense transgenic plants. Science 286, 1729-1731.

Stone, S.L., Anderson, E.M., Mullen, R.T., and Goring, D.R. (2003). $\mathrm{ARC} 1$ is an E3 ubiquitin ligase and promotes the ubiquitination of proteins during the rejection of self-incompatible Brassica pollen. Plant Cell 15, 885-898.

Stuhrwohldt, N., Dahlke, R.I., Kutschmar, A., Peng, X., Sun, M.X., and Sauter, M. (2015). Phytosulfokine peptide signaling controls pollen tube growth and funicular pollen tube guidance in Arabidopsis thaliana. Physiol Plant 153, 643-653. 
Sun, Y., Li, L., Macho, A.P., Han, Z., Hu, Z., Zipfel, C., Zhou, J.-M., and Chai, J. (2013). Structural basis for flg22-induced activation of the Arabidopsis FLS2-BAK1 immune complex. Science 342, 624-628.

Suzuki, G., Kai, N., Hirose, T., Fukui, K., Nishio, T., Takayama, S., Isogai, A., Watanabe, M., and Hinata, K. (1999). Genomic organization of the S locus: Identification and characterization of genes in SLG/SRK region of $\mathrm{S}(9)$ haplotype of Brassica campestris (syn. rapa). Genetics $153,391-400$.

Takasaki, T., Hatakeyama, K., Suzuki, G., Watanabe, M., Isogai, A., and Hinata, K. (2000). The $S$ receptor kinase determines self-incompatibility in Brassica stigma. Nature 403, 913-916.

Takayama, S., Shimosato, H., Shiba, H., Funato, M., Che, F.S., Watanabe, M., Iwano, M., and Isogai, A. (2001). Direct ligand-receptor complex interaction controls Brassica self-incompatibility. Nature 413, 534-538.

Takeuchi, H., and Higashiyama, T. (2012). A species-specific cluster of defensin-like genes encodes diffusible pollen tube attractants in Arabidopsis. PLoS Biol 10, e1001449.

Takeuchi, H., and Higashiyama, T. (2016). Tip-localized receptors control pollen tube growth and LURE sensing in Arabidopsis. Nature 531, 245-248.

Tang, W., Ezcurra, I., Muschietti, J., and McCormick, S. (2002). A cysteine-rich extracellular protein, LAT52, interacts with the extracellular domain of the pollen receptor kinase LePRK2. Plant Cell 14, 2277-2287.

Tang, W., Kelley, D., Ezcurra, I., Cotter, R., and McCormick, S. (2004). LeSTIG1, an extracellular binding partner for the pollen receptor kinases LePRK1 and LePRK2, promotes pollen tube growth in vitro. Plant J 39, 343-353.

Tsukamoto, T., Qin, Y., Huang, Y., Dunatunga, D., and Palanivelu, R. (2010). A role for LORELEI, a putative glycosylphosphatidylinositolanchored protein, in Arabidopsis thaliana double fertilization and early seed development. Plant J 62, 571-588.

Wang, J., Li, H., Han, Z., Zhang, H., Wang, T., Lin, G., Chang, J., Yang, W., and Chai, J. (2015). Allosteric receptor activation by the plant peptide hormone phytosulfokine. Nature 525, 265-268.
Wang, T., Liang, L., Xue, Y., Jia, P.F., Chen, W., Zhang, M.X., Wang, Y.C., Li, H.J., and Yang, W.C. (2016). A receptor heteromer mediates the male perception of female attractants in plants. Nature 531, 241-244.

Wengier, D., Valsecchi, I., Cabanas, M.L., Tang, W.H., McCormick, S., and Muschietti, J. (2003). The receptor kinases LePRK1 and LePRK2 associate in pollen and when expressed in yeast, but dissociate in the presence of style extract. Proc Natl Acad Sci USA 100, 6860-6865.

Wengier, D.L., Mazzella, M.A., Salem, T.M., McCormick, S., and Muschietti, J.P. (2010). STIL, a peculiar molecule from styles, specifically dephosphorylates the pollen receptor kinase LePRK2 and stimulates pollen tube growth in vitro. BMC Plant Biol 10, 33 .

Wolf, S., and Greiner, S. (2012). Growth control by cell wall pectins. Protoplasma 249 Suppl 2, S169-S175.

Wuest, S.E., Vijverberg, K., Schmidt, A., Weiss, M., Gheyselinck, J., Lohr, M., Wellmer, F., Rahnenführer, J., von Mering, C., and Grossniklaus, U. (2010). Arabidopsis female gametophyte gene expression map reveals similarities between plant and animal gametes. Curr Biol 20, 506-512.

Yalovsky, S., Bloch, D., Sorek, N., and Kost, B. (2008). Regulation of membrane trafficking, cytoskeleton dynamics, and cell polarity by ROP/RAC GTPases. Plant Physiol 147, 1527-1543.

Yang, W.-C., Shi, D.-Q., and Chen, Y.-H. (2010). Female gametophyte development in flowering plants. Annu Rev Plant Biol 61, 89-108.

Zhang, D., Wengier, D., Shuai, B., Gui, C.P., Muschietti, J., McCormick, S., and Tang, W.H. (2008). The pollen receptor kinase LePRK2 mediates growth-promoting signals and positively regulates pollen germination and tube growth. Plant Physiol 148, 1368-1379.

Zhang, Y., and McCormick, S. (2007). A distinct mechanism regulating a pollen-specific guanine nucleotide exchange factor for the small GTPase Rop in Arabidopsis thaliana. Proc Natl Acad Sci USA 104, 18830-18835.

Zhang, Y., He, J., and McCormick, S. (2009). Two Arabidopsis AGC kinases are critical for the polarized growth of pollen tubes. Plant J 58, 474-484.

Open Access This article is distributed under the terms of the Creative Commons Attribution License which permits any use, distribution, and reproduction in any medium, provided the original author(s) and source are credited. 